Lionel Pournin ${ }^{1}$

\title{
A result on flip-graph connectivity
}

June 21, 2011

\begin{abstract}
A polyhedral subdivision of a $d$-dimensional point configuration $\mathcal{A}$ is $k$-regular if it is projected from the boundary complex of a polytope with dimension at most $d+k$. Call $\gamma_{k}(\mathcal{A})$ the subgraph induced by $k$-regular triangulations in the flip-graph of $\mathcal{A}$. Gel'fand, Kapranov, and Zelevinsky have shown that $\gamma_{1}(\mathcal{A})$ is connected. It is established here that $\gamma_{2}(\mathcal{A})$ is connected as well.
\end{abstract}

\section{Flip-graph connectivity and regular subdivisions}

Let $\mathcal{A}$ be a $d$-dimensional point configuration, that is a finite subset of $\mathbb{R}^{n}$ whose affine hull has dimension $d$. A polyhedral subdivision of $\mathcal{A}$ is a collection $S$ of subsets of $\mathcal{A}$ so that $\{\operatorname{conv}(s): s \in S\}$ is a polyhedral complex and $\cup_{s \in S} \operatorname{conv}(s)$ is exactly $\operatorname{conv}(\mathcal{A})$. In this paper, the set $\omega(\mathcal{A})$ of all subdivisions of $\mathcal{A}$ will be partially ordered by the following refinement relation: a subdivision $S$ refines another subdivision $S^{\prime}$ if every face of $S$ is a subset of some face of $S^{\prime}$.

A first remarkable subposet of $\omega(\mathcal{A})$ is the flip-graph of $\mathcal{A}$, made up of the minimal and next-to-minimal elements of $\omega(\mathcal{A})$, and denoted by $\gamma(\mathcal{A})$ in the following. Observe that the minimal elements in $\omega(\mathcal{A})$ are precisely the triangulations of $\mathcal{A}$. Its next-to-minimal elements will be called flips according to the definition found in [19] (definition 4.7.) and [21] (remark 1.17.). This definition identifies flips within the refinement poset of a point configuration, but other equivalent definitions are possible $[18,19,21]$. In particular, it is more usual to define flips not as subdivisions, but as local operations instead that transform a triangulation of a point configuration into another one. The flip-graph of a point configuration $\mathcal{A}$ then is the graph whose vertices are the triangulations of $\mathcal{A}$ and whose edges are its flips. While this definition is equivalent to the one above [18,21], it better shows that flips are also used to build triangulations incrementally [8,12].

The connectivity of the flip-graph then arises as a natural question: is it always possible to transform a triangulation of a point configuration into

\footnotetext{
${ }^{1}$ Mathematics Institute, Ecole Polytechnique Fédérale de Lausanne, 1015 Lausanne, Switzerland.

Present address: EFREI, 30-32 avenue de la République, 94800 Villejuif, France e-mail: lionel.pournin@efrei.fr
} 
another one using a sequence of flips? While 2-dimensional point configurations have connected flip-graphs [11], point configurations of dimensions five and six with disconnected flip-graphs have been found $[6,18,20,21]$. It has also been shown that the flip-graph of $d$-dimensional configurations with at most $d+4$ points are necessarily connected [2]. The connectivity status of the flip-graph remains an open problem in general in dimensions 3 and 4 .

A second remarkable subposet of $\omega(\mathcal{A})$ is made up of the regular subdivisions of $\mathcal{A}$. Those subdivisions can be defined using height functions, that is any map from $\mathcal{A}$ into $\mathbb{R}$ : a polyhedral subdivision $S \subset \omega(\mathcal{A})$ is regular if there exists a height function $w: \mathcal{A} \rightarrow \mathbb{R}$ so that for all $s \in S$, there is an affine map $\xi: \mathbb{R}^{n} \rightarrow \mathbb{R}$ that coincides with $w$ on $s$ and that further satisfies $\xi(a)<w(a)$ for all $a \in \mathcal{A} \backslash s$. Such height functions $w$ are said to realize $S$. This construction actually amounts to lift a $d$-dimensional point configuration into $\mathbb{R}^{d+1}$ using a height function in order to obtain a polytope in $\mathbb{R}^{d+1}$ whose lower faces are then projected back into $\mathbb{R}^{d}$. Following that, a height function $w: \mathcal{A} \rightarrow \mathbb{R}$ realizes a unique polyhedral subdivision of $\mathcal{A}$ that will be denoted by $r(w)$ in the following of this paper. The subposet of $\omega(\mathcal{A})$ whose elements are the regular subdivisions of $\mathcal{A}$ will be referred to as $\rho(\mathcal{A})$. It has been proven in $[9,10]$ that $\rho(\mathcal{A})$ is isomorphic to the face lattice of the secondary polytope. As a consequence, the subgraph induced by regular triangulations in $\gamma(\mathcal{A})$ is connected.

In section 2 of this paper, a classification of all polyhedral subdivisions is first introduced that generalizes the construction of regular subdivisions: a polyhedral subdivision of a $d$-dimensional point configuration is $k$-regular if it is projected from the boundary complex of a polytope with dimension at most $d+k$. The subgraph induced by the $k$-regular triangulations in the flip-graph of $\mathcal{A}$ will be denoted by $\gamma_{k}(\mathcal{A})$. As mentioned above, Gel'fand, Kapranov, and Zelevinsky have shown in $[9,10]$ that $\gamma_{1}(\mathcal{A})$ is connected. It is established at the end of section 2 that $\gamma_{2}(\mathcal{A})$ is connected as well. In section 3 , several non-regular subdivisions known from the literature in dimensions 2 and 3 are studied. They all turn out to be 2-regular. Note in particular that no subdivision is known so far in dimension less than 5 that is not 2-regular. Six of the studied examples are triangulations, proving that $\gamma_{1}(\mathcal{A})$ is indeed a proper subgraph of $\gamma_{2}(\mathcal{A})$. It is shown in addition that 2-regular subdivisions are not necessarily shellable. Those results suggest that $\gamma_{2}(\mathcal{A})$ is actually much larger than $\gamma_{1}(\mathcal{A})$. In section 4 , a class of subdivisions that contains all the examples of section 3 is formally defined and investigated. In particular, upper bounds are given on the smallest value of $k$ so that all those subdivisions are $k$-regular. Finally, open questions regarding $k$-regularity, flip-graph connectivity, and shellability are formulated in section 5, along with a conjecture.

\section{A classification of polyhedral subdivisions}

Consider a polytope $p \subset \mathbb{R}^{n}$ and an affine map $\pi: \mathbb{R}^{n} \rightarrow \mathbb{R}^{d}$. In the following of the paper, the pair $(p, \pi)$ is called a polytope projection if the restriction of 
$\pi$ to the vertex set of $p$ is injective. Let $(p, \pi)$ be a polytope projection. Call $\mathcal{V}$ the vertex set of $p$ and $\phi: \pi(\mathcal{V}) \rightarrow \mathcal{V}$ the map so that $\pi \circ \phi$ is the identity. A polyhedral subdivision $S$ is induced by $\pi$ from $p$ if it is a subdivision of $\pi(\mathcal{V})$ so that for all $s \in S$, the convex hull of $\phi(s)$ is a face of $p$. The poset $\omega(p, \pi)$ of all polyhedral subdivisions induced by $\pi$ from $p$, partially ordered by the refinement relation was introduced in $[4,5]$. Observe that in $[4,5,15]$, the elements of $\omega(p, \pi)$ could be subdivisions of multisets. This more general framework is not needed here, though. The following definition introduces a classification of polyhedral subdivisions:

Definition 1. A subdivision $S$ is called $k$-regular if there exists a polytope projection $(p, \pi)$ so that $S \in \omega(p, \pi)$ and $\operatorname{dim}(p)-\operatorname{dim}(\pi(p)) \leq k$.

Let $\mathcal{A}$ be a point configuration. The subposet of $\omega(\mathcal{A})$ whose elements are the $k$-regular subdivisions of $\mathcal{A}$ will be denoted by $\rho_{k}(\mathcal{A})$. According to Definition $1,\left(\rho_{k}(\mathcal{A})\right)_{k \geq 0}$ is an increasing sequence of posets whose largest elements are identically equal to $\omega(\mathcal{A})$. Indeed, calling $n$ the cardinality of $\mathcal{A}$ and $d$ the dimension of its affine hull, there exists a simplex in $\mathbb{R}^{n-1}$ whose vertex set projects onto $\mathcal{A}$. It follows that $\rho_{k}(\mathcal{A})=\omega(\mathcal{A})$ whenever $k \geq n-d-1$. Poset $\rho_{0}(\mathcal{A})$ is a singleton whose unique element is the trivial subdivision of $\mathcal{A}$. This subdivision, denoted by $t(\mathcal{A})$ in the following, is the only subdivision of $\mathcal{A}$ that admits $\mathcal{A}$ itself as a face. Posets $\rho(\mathcal{A})$ and $\rho_{1}(\mathcal{A})$ are identical:

Proposition 1. A subdivision is regular if and only if it is 1-regular.

Proof. Let $\mathcal{A} \subset \mathbb{R}^{d}$ be a $d$-dimensional point configuration and $S$ a subdivision of $\mathcal{A}$. It will be assumed here that $\mathcal{A}$ is actually the vertex set of $S$. Throughout this proof, $\mathbb{R}^{d}$ is identified with a linear subspace of $\mathbb{R}^{d+1}$ and $u \in \mathbb{R}^{d+1}$ denotes a unit vector orthogonal to $\mathbb{R}^{d}$.

First assume that $S$ is regular. Let $w$ be a height function that realizes $S$. Denote by $\pi$ the orthogonal projection from $\mathbb{R}^{d+1}$ onto $\mathbb{R}^{d}$. For all $a \in \mathcal{A}$, call $\phi(a)=a+w(a) u$ and denote $p=\operatorname{conv}(\phi(\mathcal{A}))$. Observe that $(p, \pi)$ is a polytope projection. Now let $s$ be any face of $S$. As $w$ realizes $S$, there exists an affine map $\xi: \mathbb{R}^{d} \rightarrow \mathbb{R}$ that coincides with $w$ on $s$ and satisfies $\xi(a)<w(a)$ for all $a \notin s$. Consider the affine map $\psi: \mathbb{R}^{d+1} \rightarrow \mathbb{R}$ so that for all $x \in \mathbb{R}^{d+1}, \psi(x)=\xi \circ \pi(x)-x . u$. Following this, $\psi \circ \phi$ maps $s$ onto $\{0\}$ and $\mathcal{A} \backslash s$ into $]-\infty, 0[$. The convex hull of $\phi(s)$ then is a face of $p$, proving that $S \in \omega(p, \pi)$ and, as $\operatorname{dim}(p)=\operatorname{dim}(\pi(p))+1$, that $S$ is 1-regular.

Now assume that $S$ is 1-regular. Let $(p, \pi)$ be a polytope projection so that $S \in \omega(p, \pi)$ and $p$ is $(d+1)$-dimensional. One can assume without loss of generality that $\pi$ is the orthogonal projection from $\mathbb{R}^{d+1}$ onto $\mathbb{R}^{d}$. Call $\mathcal{V}$ the vertex set of $p$ and $\phi: \pi(\mathcal{V}) \rightarrow \mathcal{V}$ the map so that $\pi \circ \phi$ is the identity. Since $S \in \omega(p, \pi)$, then for any $s \in S$, the convex hull of $\phi(s)$ is a face of $p$ and the normal cone $C_{s}$ of $p$ at $\operatorname{conv}(\phi(s))$ is thereofore non-empty.

Let $s$ and $s^{\prime}$ be two $d$-dimensional faces of $S$. Let $y \in C_{s}$ and $y^{\prime} \in C_{s^{\prime}}$. It is proven in this paragraph that $y . u$ and $y^{\prime} \cdot u$ are both positive or both 
negative. First observe that $y . u$ and $y^{\prime} . u$ are necessarily non-zero (otherwise the dimension of $s$ would at most be $d-1$ ). Next, assume that $s$ and $s^{\prime}$ admit a common $(d-1)$-dimensional face $f$. If $y . u$ and $y^{\prime} . u$ have opposite signs, then by convexity of cone $C_{f}$ there necessarily exists a vector $z \in C_{f}$ so that $z . u=0$. As a consequence, $z$ also belongs to the normal cone of $\pi(p)$ at $\operatorname{conv}(f)$. As $\operatorname{conv}(f)$ is not a face of $\pi(p)$ (otherwise it would be incident to only one $d$-dimensional face of $S$ ), a contradiction is obtained, showing that $y . u$ and $y^{\prime} . u$ are either both positive or both negative. By connectivity of the dual graph of $S$ (i. e. the graph whose vertices are the $d$-dimensional faces of $S$ and whose edges connect the faces that share a common proper $(d-1)$-dimensional face), this property finally carries over to any pair $\left(s, s^{\prime}\right)$ of $d$-dimensional faces of $S$. One therefore obtains, possibly by negating $u$, that for any $d$-dimensional face $s$ of $S$, there exists $y \in C_{s}$ so that $y . u=1$.

For every vertex $a$ of $S$, call $w(a)=\phi(a)$.u. Let $s$ be a $d$-dimensional face of $S$ and $y$ a vector in $C_{s}$ so that $y . u=1$. As $y \in C_{s}$, there exists $m \in \mathbb{R}$ so that for all $a \in s, \phi(a) . y=m$ and for all $a \in \mathcal{A} \backslash s, \phi(a) . y<m$. Consider the affine map $\xi: \mathbb{R}^{d} \rightarrow \mathbb{R}$ so that for all $x \in \mathbb{R}^{d}, \xi(x)=x \cdot y-m$. As $y . u=1$, then $\xi$ coincides with $w$ on $s$ and satisfies $\xi(a)<w(a)$ for all $a \notin s$. All the $d$-dimensional faces of $S$ therefore belong to $r(w)$ and as a consequence, $S=r(w)$ which finishes the proof.

Observe that the graph $\gamma_{k}(\mathcal{A})$ introduced in section 1 can be identified with the intersection of posets $\rho_{k}(\mathcal{A})$ and $\gamma(\mathcal{A})$. In other words, $\gamma_{k}(\mathcal{A})$ is the subposet of $\rho_{k}(\mathcal{A})$ that contains only the triangulations and the flips. The connectedness of $\gamma_{2}(\mathcal{A})$ will be established in the following of this section. The proof, and in particular that of theorem 1, uses results by Rambau and Ziegler [15] and Alexeev [1]. The statement of those results rely on the notion of coherence due to Billera et al. [4,5] (see also [18,19]) that alternatively generalizes regularity in the context of polytope projections.

Let $(p, \pi)$ be a polytope projection. A subdivision in $\omega(p, \pi)$ is coherent if it belongs to $\omega\left(\pi_{1}(p), \pi_{2}\right)$ for a pair $\left(\pi_{1}, \pi_{2}\right)$ of affine maps so that $\pi=\pi_{2} \circ \pi_{1}$ and $\operatorname{dim}\left(\pi_{1}(p)\right)-\operatorname{dim}(\pi(p))=1$. The subposet of $\omega(p, \pi)$ whose elements are the coherent subdivisions induced by $\pi$ from $p$ is denoted here by $\rho(p, \pi)$. It has been shown in [4] that $\rho(p, \pi)$ is isomorphic to the face lattice of the fiber polytope. While $\omega(p, \pi)$ itself is generally not isomorphic to the face lattice of a polytope, it has been conjectured that the inclusion map $\rho(p, \pi) \hookrightarrow \omega(p, \pi)$ is a homotopy equivalence [3,5]. This conjecture, called generalized Baues conjecture, has been proven false in general and true if $\operatorname{dim}(p) \leq \operatorname{dim}(\pi(p))+2[15]$. The latter result will be used in the proof of theorem 1 .

The notion of coherent refinement, due to Alexeev [1] will be needed as well. Let $S$ be a polyhedral subdivision. A system of height functions on $S$ is a family $\left(w_{s}\right)_{s \in S}$ so that for all $s \in S, w_{s}$ is a height function on $s$, and for all $\left(s, s^{\prime}\right) \in S^{2}, w_{s}$ and $w_{s^{\prime}}$ differ by an affine map on $s \cap s^{\prime}$. A refinement $S^{\prime}$ of $S$ is said to coherently refine $S$ if there is a system of height functions $\left(w_{s}\right)_{s \in S}$ so that for all $s \in S, r\left(w_{s}\right) \subset S^{\prime}$. If $S$ is a 
polyhedral subdivision of a point configuration $\mathcal{A}$, denote by $\rho(\mathcal{A}, S)$ the subposet of $\omega(\mathcal{A})$ whose elements are the coherent refinements of $S$. It is shown in [1] (lemma 2.12.11.) that $\rho(\mathcal{A}, S)$ is isomorphic to the face lattice of the generalized secondary polytope. This result will be invoked in the proof of theorem 1.

The following first lemma shows that a triangulation induced from a polytope is always induced from a simplicial polytope of the same dimension:

Lemma 1. Let $\mathcal{A}$ be a point configuration and $T$ a triangulation of $\mathcal{A}$. If $T$ is $k$-regular, there necessarily exists a polytope projection $(p, \pi)$ that satisfies the following statements:

i. $T \in \omega(p, \pi)$,

ii. $\operatorname{dim}(p)-\operatorname{dim}(\pi(p)) \leq k$,

iii. $\omega(p, \pi) \subset \omega(\mathcal{A})$,

iv. $p$ is simplicial.

Proof. According to definition 1, there exists a polytope projection $(q, \pi)$ so that $T \in \omega(q, \pi)$ and $\operatorname{dim}(q)-\operatorname{dim}(\pi(q)) \leq k$. Consider the vertices of $q$ whose images by $\pi$ are vertices of $T$ and call $p$ their convex hull. Polytope projection $(p, \pi)$ then satisfies statements $i$., ii., and iii. above.

Let $\mathcal{V}$ denote the vertex set of $p$. The cardinality of $\mathcal{V}$ and the affine dimension of its image by $\pi$ will be respectively denoted by $n$ and $d$. The set $L$ of all maps $\lambda: \pi(\mathcal{V}) \rightarrow \mathbb{R}^{d+k}$ so that $\pi \circ \lambda$ is the identity can be identified with $\mathbb{R}^{k n}$. For any $\lambda \in L$, call $p_{\lambda}$ the convex hull of $\lambda \circ \pi(\mathcal{V})$, and consider the set $M=\left\{\lambda \in L: T \in \omega\left(p_{\lambda}, \pi\right)\right\}$. Let $\phi: \pi(\mathcal{V}) \rightarrow \mathcal{V}$ be the map so that $\pi \circ \phi$ is the identity. As $T$ is induced by $\pi$ from $p$, then $\phi$ is naturally an element of $M$. Moreover, as $T$ is a triangulation, $M$ has dimension $k n$. Now consider the set $N$ of all maps $\lambda \in L$ so that $p_{\lambda}$ is not simplicial. This set is the union of finitely many affine cones, and each of these cones has dimension at most $k n-1$. As a consequence, there necessarily exists some $\mu \in M \backslash N$. Polytope projection $\left(p_{\mu}, \pi\right)$ then satisfies the four above statements, which proves the lemma.

The main result of this paper will be obtained considering polytope projections $(p, \pi)$ so that $p$ is simplicial and $\operatorname{dim}(p)-\operatorname{dim}(\pi(p)) \leq 2$. The following lemma states two properties of such polytope projections that will be invoked in the coming proofs. The statement of the first property mentions circuits. Those objects arise in the theory of matroids: a circuit is an affinely dependent set that is minimal for the inclusion.

Lemma 2. Let $(p, \pi)$ be a polytope projection and $S$ a non maximal element of $\omega(p, \pi)$. If $p$ is simplicial and $\operatorname{dim}(p)-\operatorname{dim}(\pi(p)) \leq 2$ then:

i. Every face of $S$ contains at most one circuit,

ii. Every refinement of $S$ belongs to $\omega(p, \pi)$.

Proof. Denote $d=\operatorname{dim}(\pi(p))$ and let $s$ be a face of $S$. There exists some $d$-dimensional face $s^{\prime}$ of $S$ so that $s \subset s^{\prime}$. Since $S$ is not maximal in $\omega(p, \pi)$, 
there is a proper face of $p$ whose vertex set is projected onto $s^{\prime}$ by $\pi$. As $p$ is $\operatorname{simplicial}$ and $\operatorname{dim}(p) \leq d+2, s^{\prime}$ has at most $d+2$ vertices.

As $s^{\prime}$ is $d$-dimensional, it contains an affinely independent subset of $d+1$ elements. If $s^{\prime}$ has $d+1$ vertices then as a simplex it does not admit any affinely dependent subset. If $s^{\prime}$ has $d+2$ vertices then there exists a unique affine dependence between them. In other words, $s^{\prime}$ admits exactly one affinely dependent subset (obtained as the set of its elements with non-zero coefficients in its affine dependence). As a consequence $s$ admits at most one circuit as a subset and $i$. is proven.

Call $f$ the proper face of $p$ whose vertex set is projected onto $s$ by $\pi$. Since $f$ is a simplex, the convex hull of any subset of $s$ is the image by $\pi$ of a face of $s$. As a consequence, any refinement of $S$ belongs to $\omega(p, \pi)$.

In order to state the next theorems, some definition and properties first have do be given. Let $\mathcal{A}$ be a point configuration. The definition of flips as next-to-minimal elements in $\omega(\mathcal{A})$ is elegant but not very practical. The following proposition (that combines lemma 4.2. in [18] and lemma 1.12. in [21]) provides alternative characterizations of flips:

Proposition 2. Let $S$ be a polyhedral subdivision of a point configuration $\mathcal{A}$. The following statements are equivalent:

i. $S$ is a next-to-minimal element of $\omega(\mathcal{A})$,

ii. There is a unique circuit contained in some face of $S$,

iii. $S$ admits exactly two proper refinements,

iv. All the proper refinements of $S$ are triangulations.

This proposition states in particular that a flip is a polyhedral subdivision that admits two triangulations as its only proper refinements. The implication from point $i$. to points $i i i$. and $i v$. in proposition 2 is obtained invoking the following consequence of Radon's partition theorem [14]: a circuit $z$ admits exactly two subdivisions other than $t(z)$. Now observe that if $S$ is a flip and $z$ is the circuit contained in some of its faces, then each refinement of $S$ admits one of the three subdivisions of $z$ as a subset. The following proposition will be used in the proof of lemma 3 :

Proposition 3. Let $S$ be a flip and $z$ the circuit contained in some of its faces. Two refinements of $S$ that admit the same subdivision of $z$ as a common subset are necessarily identical.

Note that the following lemma is independent of lemmas 1 and 2. It states that under some conditions, all the refinements of a subdivision coherently refine it.

Lemma 3. Let $\mathcal{A}$ be a point configuration, and $S \in \omega(\mathcal{A}) \backslash \gamma(\mathcal{A})$. If every face of $S$ admits at most one affinely dependent subset, then all the refinements of $S$ belong to $\rho(\mathcal{A}, S)$. 
Proof. Let $S^{\prime}$ be a refinement of $S$ and $s$ a face of $S$. Denote by $G(s)$ the subdivision of $s$ found as a subset of $S^{\prime}$. Observe that $s$ either is a simplex, or a circuit, or it admits exactly one circuit as a proper subset. If $s$ is a simplex then $G(s)=t(s)$. In this case $G(s)$ is regular and there exists a height function $w_{s}: s \rightarrow \mathbb{R}$ that realizes $G(s)$. If $s$ is a circuit, all its subdivisions are regular and there is a height function $w_{s}: s \rightarrow \mathbb{R}$ so that $G(s)=r\left(w_{s}\right)$. Now assume $s$ admits exactly one circuit $z$ as a proper subset. This circuit is necessarily a face of $s$, otherwise $s$ would have at least two affinely independent subsets. As a consequence $z \in S$. Observe that $G(z) \subset G(s)$. Let $w_{s}: s \rightarrow \mathbb{R}$ be a height function that coincides with $w_{z}$ on $z$. Observe that $r\left(w_{z}\right)$ is naturally a subset of $r\left(w_{s}\right)$. Since $t(s)$ is a flip and $z$ is the unique circuit possibly found as a subset of its faces, then two refinements of $t(s)$ that admit the same subdivision of $z$ as a subset are necessarily identical, according to proposition 3. Since $r\left(w_{s}\right)$ and $G(s)$ both admit $G(z)$ a a subset then $r\left(w_{s}\right)=G(s)$, which shows that for all $s \in S$, $r\left(w_{s}\right) \subset S^{\prime}$.

Finally, the family $\left(w_{s}\right)_{s \in S}$ is a system of height functions on $S$. Indeed if $s$ and $s^{\prime}$ are two faces of $S$, their intersection is either a circuit or a simplex. If $s \cap s^{\prime}$ is a circuit, then both $w_{s}$ and $w_{s^{\prime}}$ are equal to $w_{s \cap s^{\prime}}$ on $s \cap s^{\prime}$. If $s \cap s^{\prime}$ is a simplex then $w_{s}$ and $w_{s^{\prime}}$ obviously differ by an affine map on $s \cap s^{\prime}$. This proves that any refinement of $S$ coherently refines $S$.

For a polytope projection $(p, \pi)$, the subposet of $\omega(p, \pi)$ whose elements are the triangulations and the flips induced by $\pi$ from $p$ will be denoted by $\gamma(p, \pi)$ in the following.

Theorem 1. If $(p, \pi)$ is a polytope projection so that $p$ is simplicial and $\operatorname{dim}(p)-\operatorname{dim}(\pi(p)) \leq 2$ then $\gamma(p, \pi)$ is connected.

Proof. Let $S$ and $S^{\prime}$ be two elements of $\gamma(p, \pi)$. Both $S$ and $S^{\prime}$ then belong to $\omega(p, \pi)$. As $\operatorname{dim}(p)-\operatorname{dim}(\pi(p)) \leq 2$, the inclusion map $\rho(p, \pi) \hookrightarrow \omega(p, \pi)$ is a homotopy equivalence (see Theorem 1.6 in [15]). As $\rho(p, \pi)$ is isomorphic to the face lattice of the fiber polytope, the poset obtained by removing its largest element from $\omega(p, \pi)$ is necessarily connected. This yields a sequence $\left(S_{i}\right)_{i \in\{1, \ldots, k\}}$ of non-maximal elements of $\omega(p, \pi)$ so that $S_{1}=S, S_{k}=S^{\prime}$, and for all $i \in\{2, \ldots, k\}$, either $S_{i-1}$ refines $S_{i}$ or $S_{i}$ refines $S_{i-1}$.

It is proven by induction on $k$ that $S$ and $S^{\prime}$ are connected by elements of $\gamma(p, \pi)$. If $k \leq 2$, the result is obvious. Now if $k=3$, call $\mathcal{A}$ the image by $\pi$ of the vertex set of $p$. Assume that $S_{2} \notin \gamma(p, \pi)$. In this case, $S$ and $S^{\prime}$ necessarily refine $S_{2}$ and as a consequence of lemmas 2 and 3 they both belong to $\rho\left(\mathcal{A}, S_{2}\right)$. As $\rho\left(\mathcal{A}, S_{2}\right)$ is isomorphic to the face lattice of the generalized secondary polytope, poset $\gamma(\mathcal{A}) \cap \rho\left(\mathcal{A}, S_{2}\right)$ is isomorphic to the 1-skeleton of this polytope and is therefore connected. Since $S$ and $S^{\prime}$ both belong to $\gamma(\mathcal{A}) \cap \rho\left(\mathcal{A}, S_{2}\right)$, they are connected by a sequence of its elements. According to statement $i$. in lemma $2, \rho\left(\mathcal{A}, S_{2}\right)$ is a subset of $\omega(p, \pi)$, which proves that $S$ and $S^{\prime}$ are connected in $\gamma(p, \pi)$. If $k>3$ there is a triangulation $T$ that refines both $S_{2}$ and $S_{3}$. This breaks the 
sequence $\left(S_{i}\right)_{i \in\{1, \ldots, k\}}$ into two smaller sequences: $S_{1}, S_{2}, T$ and $T, S_{3}, \ldots$, $S_{k}$. According to statement $i$. in lemma $2, T \in \gamma(p, \pi)$. By induction, $S$ is connected to $T$ and $T$ is connected to $S^{\prime}$ by elements of $\gamma(p, \pi)$.

The following theorem is the main result of this article. It is proven by invoking lemma 1 and theorem 1 .

Theorem 2. For any point configuration $\mathcal{A}, \gamma_{2}(\mathcal{A})$ is connected.

Proof. Let $T$ be a 2-regular triangulation of $\mathcal{A}$. According to lemma 1 , there exists a polytope projection $(p, \pi)$ so that $T$ is induced by $\pi$ from $p, \omega(p, \pi)$ is a subset of $\omega(A), p$ is simplicial, and $\operatorname{dim}(p)-\operatorname{dim}(\pi(p)) \leq 2$.

As $p$ is simplicial the minimal elements in $\rho(p, \pi)$ are triangulations. Moreover, $\rho(p, \pi)$ being a subposet of $\rho(\mathcal{A})$, those triangulations are necessarily regular. Poset $\omega(p, \pi)$ hence contains some regular triangulation of $\mathcal{A}$. According to theorem 1, $T$ is connected to this triangulation by elements of $\gamma(p, \pi)$. As both $\gamma_{1}(\mathcal{A})$ and $\gamma(p, \pi)$ are subposets of $\gamma_{2}(\mathcal{A})$, the result follows from the connectivity of $\gamma_{1}(\mathcal{A})$.

\section{Examples}

Several examples of non-regular subdivisions can be found in the mathematical literature. The most popular among them is the 2-dimensional triangulation depicted in the upper-left part of Fig. 1 and referred to as example (I) in the following. This triangulation of a configuration of 6 points is obtained by looking inside Schönhardt's polyhedron [22] from above. Several such non-regular examples are studied in this section and proven 2-regular. This result is established in particular for Rudin's triangulation [17], showing that 2-regular subdivisions are not necessarily shellable.

Observe that triangulation (I) is obtained by connecting the boundary faces of two nested triangles. This construction can be generalized to arbitrary large point configurations just as Schönhardt's polyhedron was generalized in [16]. Subdivisions (II) and (III) shown in Fig. 1 are built this way from, respectively, two nested squares and two nested pentagons. This construction ends up in general with non-regular subdivisions. One can prove that subdivisions (I), (II), and (III) are non-regular by invoking the criterion formulated in [7]: they all admit a cycle of overlapping faces with respect to the point in their center. In triangulation (I), the cycle is made up of the edges $\{1,5\},\{2,6\}$, and $\{3,4\}$. One can check that these edges indeed form a cycle as defined in [7] by looking in Fig. 1 at the dotted lines that sketch the affine spans of edges $\{1,4\},\{2,5\}$, and $\{3,6\}$. In subdivision (II) the cycle is made up of edges $\{1,6\},\{2,7\},\{3,8\}$, and $\{4,5\}$. In the case of (III), edges $\{1,7\},\{2,8\},\{3,9\},\{4,10\}$, and $\{5,6\}$ form a cycle of the same kind. This shows that (I), (II), and (III) are non-regular. Those three examples turn out to be 2-regular, though. In order to prove it, a theorem is needed that will now be stated. 
Let $S$ be a subdivision. The smallest integer $k$ such that $S$ is $k$-regular will be denoted by $\delta(S)$. The following theorem is a first convenient way to find upper bounds on $\delta(S)$.

Theorem 3. Let $\mathcal{A}$ be a point configuration and $\left(S_{i}\right)_{0 \leq i \leq n}$ a finite family of subdivisions of $\mathcal{A}$. If $S_{0} \subset \cup_{i=1}^{n} S_{i}$ then $\delta\left(S_{0}\right) \leq \sum_{i=1}^{n} \delta\left(S_{i}\right)$.

Proof. Call $d$ the affine dimension of $\mathcal{A}$, and assume that the affine hull of $\mathcal{A}$ is precisely $\mathbb{R}^{d}$. For all $i \in\{1, \ldots, n\}$, there exists a polytope projection $\left(p_{i}, \pi_{i}\right)$ so that $S_{i} \in \omega\left(p_{i}, \pi_{i}\right)$ and $\operatorname{dim}\left(p_{i}\right)=d+\delta\left(S_{i}\right)$. One can assume without loss of generality that $\mathbb{R}^{d}$ is identified with a linear subspace of $\mathbb{R}^{d+\delta\left(S_{i}\right)}$ and that $\pi_{i}$ is the orthogonal projection from $\mathbb{R}^{d+\delta\left(S_{i}\right)}$ onto $\mathbb{R}^{d}$. Now denote by $m$ the sum $\sum_{i=1}^{n} \delta\left(S_{i}\right)$, and identify $\mathbb{R}^{d}$ with a linear subspace of $\mathbb{R}^{d+m}$. The orthogonal complement of $\mathbb{R}^{d}$ in $\mathbb{R}^{d+m}$ can be decomposed as a direct sum $\oplus_{i=1}^{n} E_{i}$ of mutually orthogonal linear spaces $\left(E_{i}\right)_{1 \leq i \leq n}$, where for all $i \in\{1, \ldots, n\}, E_{i}$ has dimension $\delta\left(S_{i}\right)$.

For all $i \in\{1, \ldots, n\}$, let $\xi_{i}: \operatorname{ker}\left(\pi_{i}\right) \rightarrow E_{i}$ be a linear bijection. Further call $\mathcal{V}_{i}$ the vertex set of $p_{i}, \phi_{i}: \pi_{i}\left(\mathcal{V}_{i}\right) \rightarrow \mathcal{V}_{i}$ the map so that $\pi_{i} \circ \phi_{i}$ is the identity, and $\eta_{i}$ the orthogonal projection from $\mathbb{R}^{d+\delta\left(S_{i}\right)}$ onto $\operatorname{ker}\left(\pi_{i}\right)$. Let $\mathcal{A}_{0}$ be the vertex set of $S_{0}$. The map $\phi_{i}$ can be extended to the whole of $\mathcal{A}_{0}$ by choosing $\phi_{i}(a)$ into $\pi_{i}^{-1}(a) \cap p_{i}$ for all $a \in \mathcal{A}_{0} \backslash \pi_{i}\left(\mathcal{V}_{i}\right)$. For every $a \in \mathcal{A}_{0}$, call:

$$
\phi(a)=a+\sum_{i=1}^{n} \xi_{i} \circ \eta_{i} \circ \phi_{i}(a) .
$$

Let $p$ denote the convex hull of $\phi\left(\mathcal{A}_{0}\right)$ and $\pi$ the orthogonal projection from $\mathbb{R}^{d+m}$ onto $\mathbb{R}^{d}$. Observe that $(p, \pi)$ is a polytope projection. Now let $s$ be a face of $S_{0}$. There exists $i \in\{1, \ldots, n\}$ so that $s \in S_{i}$. As $S_{i} \in \omega\left(p_{i}, \pi_{i}\right)$, there exists an affine map $\psi: \mathbb{R}^{d+\delta\left(S_{i}\right)} \rightarrow \mathbb{R}$ that projects $\phi_{i}(s)$ onto $\{0\}$ and $\mathcal{V}_{i} \backslash \phi_{i}(s)$ into $]-\infty, 0\left[\right.$. Call $\theta_{i}$ the orthogonal projection from $\mathbb{R}^{d+m}$ onto $E_{i}$ and consider the affine map $\chi=\pi+\xi_{i}^{-1} \circ \theta_{i}$.

According to the construction of $\phi$, one can see that $\chi \circ \phi$ and $\phi_{i}$ are equal on $\mathcal{A}_{0}$. As an immediate consequence, $\psi \circ \chi$ projects $\phi(s)$ onto $\{0\}$ and the elements of $\phi\left(\mathcal{A}_{0} \cap \pi_{i}\left(\mathcal{V}_{i}\right) \backslash s\right)$ into $]-\infty, 0\left[\right.$. Now let $a \in \mathcal{A}_{0} \backslash \pi_{i}\left(\mathcal{V}_{i}\right)$. Point $a$ is a vertex of $S_{0}$ that does not belong to $s$ (recall that $s \subset \pi_{i}\left(\mathcal{V}_{i}\right)$ ). It follows that $a \notin \operatorname{conv}(s)$. As $\phi_{i}(a)$ was chosen within $\pi_{i}^{-1}(a) \cap p_{i}$, then $\phi_{i}(a)$ belongs to $p_{i} \backslash \operatorname{conv}(\phi(s))$. As a consequence, $\psi \circ \chi \circ \phi(a)<0$.

This shows that $\psi \circ \chi$ projects $\phi(s)$ onto $\{0\}$ and the whole of $\phi\left(\mathcal{A}_{0} \backslash s\right)$ into $]-\infty, 0[$. The convex hull of $\phi(s)$ is therefore a face of $p$, proving that $S_{0} \in \omega(p, \pi)$. As $\operatorname{dim}(p)-\operatorname{dim}(\pi(p))=\sum_{i=1}^{n} \delta\left(S_{i}\right)$, the result follows.

Now consider the triangulation obtained from (I) by flipping edge $\{1,5\}$ (i. e. by substituting edge $\{2,4\}$ in (I) for edge $\{1,5\}$ ). This is a regular triangulation that contains all the faces of (I) except $\{1,5\},\{1,2,5\}$, and $\{1,4,5\}$. Another regular triangulation is obtained from (I) by flipping edge $\{3,4\}$. This one contains all the faces of (I) except $\{3,4\},\{3,4,1\}$, and $\{3,4,6\}$. Following this, every face of (I) belongs to at least one of those two 

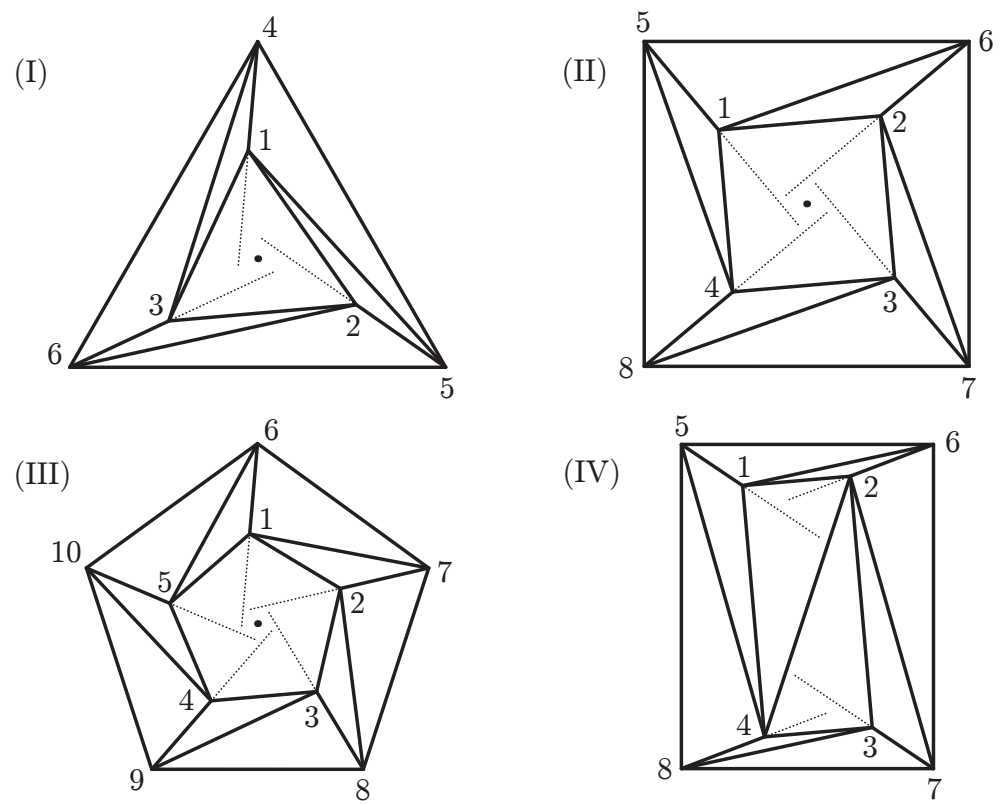

(IV)
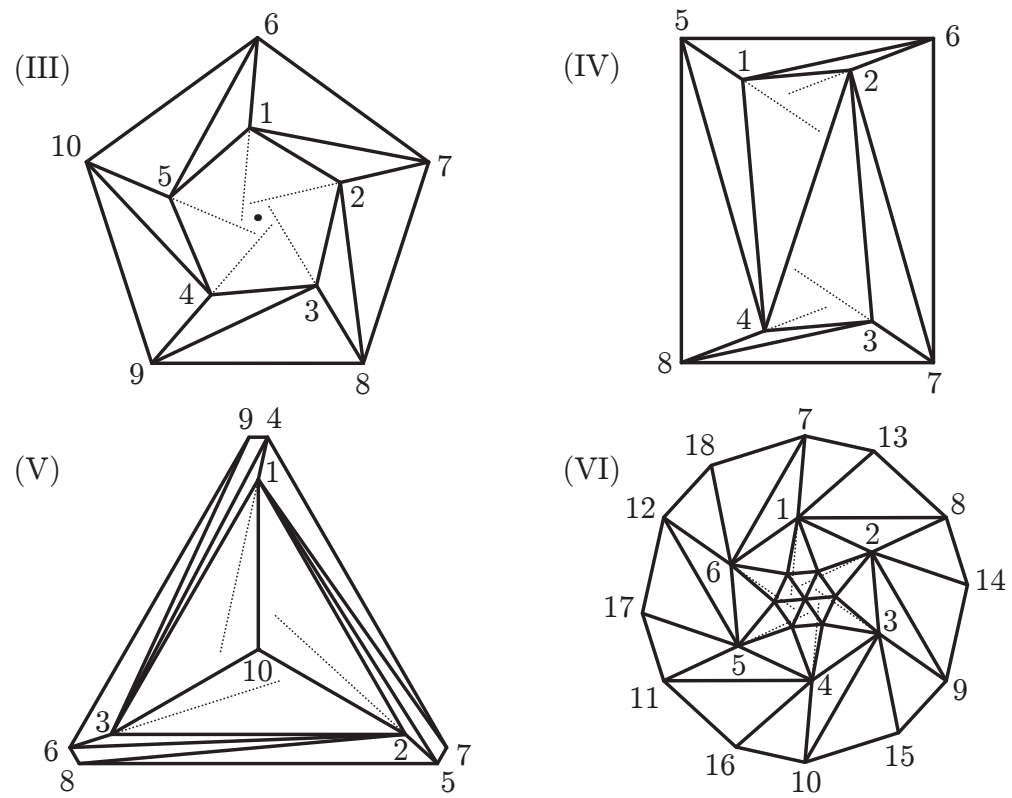

Fig. 1. Six non-regular 2-dimensional subdivisions

regular triangulations. According to theorem 3, triangulation (I) is therefore 2-regular. The same trick can be used to show that examples (II) and (III) are 2-regular: flipping edges $\{1,6\}$ and $\{4,5\}$ alternatively in subdivision (II) and edges $\{1,7\}$ and $\{5,6\}$ alternatively in subdivision (III) produces in each case two regular subdivisions whose union admit the initial subdivision as a subset. The 2-regularity of subdivisions (II) and (III) therefore also follow from theorem 3.

More complicated 2-dimensional examples are now discussed. Observe that such subdivisions as (II) and (III) can be refined by triangulating their central faces. The obtained triangulations are necessarily non-regular because they inherit the cycles of the non-regular subdivisions they are built from. Following this, the two proper refinements of (II), obtained by either inserting edge $\{1,3\}$ or edge $\{2,4\}$, are non-regular because they admit a cycle in the sense of [7]. Triangulation (IV) shown in Fig. 1 and studied in 
[23] shares its topology with the refinement of (II) that contains edge $\{2,4\}$. It is shown in [23] that despite this similarity, triangulation (IV) does not admit any cycle in the sense of [7], and that it is non-regular nonetheless. One can get convinced that edges $\{1,6\},\{2,7\},\{3,8\}$, and $\{4,5\}$ do not form a cycle in (IV) by looking in Fig. 1 at the dotted lines sketching the affine spans of edges $\{1,5\},\{2,6\},\{3,7\}$, and $\{4,8\}$. In addition, the triangulation obtained from (IV) by flipping edge $\{2,4\}$ turns out to be regular even though it shares its topology with the refinement of (II) that contain edge $\{1,3\}$. The regularity of such examples therefore depends on the way their center is triangulated, and their non-regularity does not arise from the presence of a cycle in the sense of [7]. One can prove that triangulation (IV) is 2-regular using theorem 3 in just the same way as with subdivision (II): the two triangulations obtained from (IV) by respectively flipping edge $\{1,6\}$ and edge $\{4,5\}$ are regular and their union contains all the faces of (IV). Hence, according to theorem 3, triangulation (IV) is 2-regular.

Example (V) shown in Fig. 1 is obtained from a triangulation with the same topology than (I), by connecting additional boundary vertices 7,8 , and 9 to interior vertices 1, 2, and 3 respectively. Vertex 10 is further added within the central triangle. This triangulation of a configuration of 10 points belongs to a poset described in [15] as a counter-example to the generalized Baues conjecture. Since it inherits the cycle found in triangulation (I), triangulation $(\mathrm{V})$ is non-regular. It is 2-regular, though, which is proven using theorem 3. Consider the triangulation obtained from (V) by sequentially flipping edges $\{1,7\}$ and $\{1,5\}$ in this order. This is a regular triangulation that contains all the faces of (V) except for $\{1,7\},\{1,5\},\{1,4,7\}$, $\{1,5,7\}$, and $\{1,2,5\}$. One can build a regular triangulation that contains those five missing faces by flipping edge $\{3,9\}$ first, and then edge $\{3,4\}$ in $(\mathrm{V})$. According to theorem 3, triangulation $(\mathrm{V})$ is therefore 2-regular.

Triangulation (VI), also shown in Fig. 1 is a more elaborate example of a non-regular triangulation constructed using the same general rules as for the previous five examples. It is built from two nested hexagons and thirteen additional vertices. Seven of those additional vertices are placed within the central hexagon, and the other six about the boundary of the external one. An interesting feature of this triangulation is that all of its interior edges can be flipped. While this suggests a good behavior regarding flips, it turns out that triangulation (VI) is non-regular. Indeed, edges $\{1,9\}$, $\{2,11\},\{3,13\},\{4,15\},\{5,17\}$, and $\{6,7\}$ form a cycle of overlapping faces with respect to its central point. A regular triangulation that contains all the faces of (VI) except for $\{1,8\},\{1,13\},\{1,2,8\},\{1,8,13\}$, and $\{1,7,13\}$ is found by flipping edge $\{1,13\}$ first and then edge $\{1,8\}$. Another regular triangulation that contains $\{1,8\},\{1,13\},\{1,2,8\},\{1,8,13\}$, and $\{1,7,13\}$ is obtained from (VI) by sequentially flipping edges $\{6,18\}$ and $\{6,7\}$ in this order. As a consequence, triangulation (VI) is 2-regular.

The six previous examples are 2-dimensional. A first, 3-dimensional triangulation is now studied. This triangulation, described in [13], causes usual regularization algorithms to fail. For the sake of simplicity the elements of 

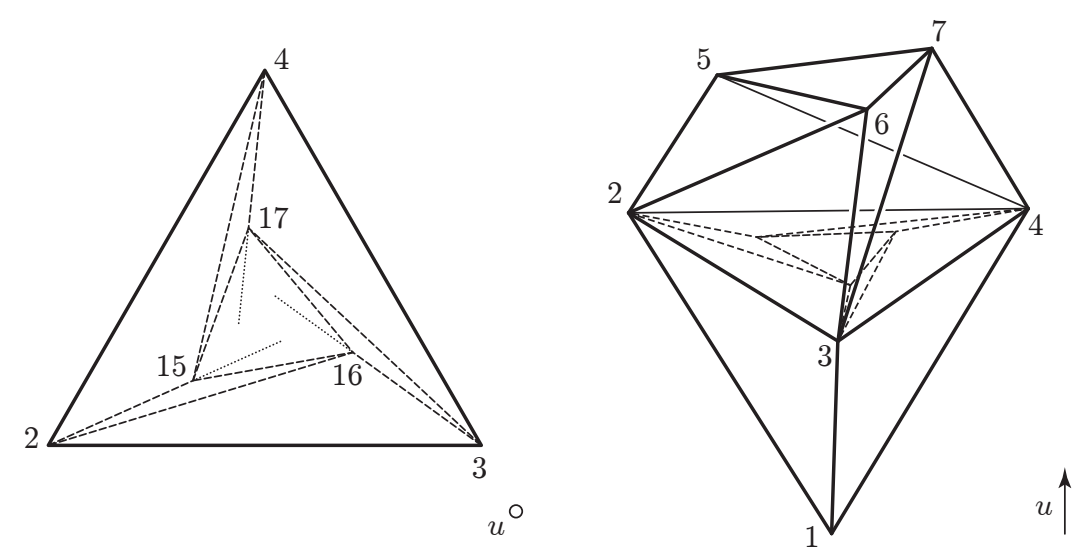

Fig. 2. Sketch of the non-regular 3-dimensional triangulation (VII)

$\mathbb{R}^{3}$ will here be identified with the vector of their coordinates along a given orthonormal basis. Let $\alpha, \beta$, and $\varepsilon$ be any solution to the following nonlinear system of inequalities:

$$
\left\{\begin{array}{l}
\alpha+2 \beta+\varepsilon>1 \\
\alpha \beta-(\beta+\varepsilon)^{2}>0 \\
\alpha, \beta, \varepsilon \quad>0
\end{array}\right.
$$

Note that such solutions exist, as for example $\alpha=5, \beta=1$, and $\varepsilon=1$. The matrix below then defines a 3-dimensional configuration of seven points denoted by $\mathcal{A}_{\text {(VII) }}$. The columns of this matrix correspond to points and its lines to coordinates:

$$
\mathcal{A}_{(\mathrm{VII})}=\left(\begin{array}{lllllll}
0 & 1 & 0 & 0 & \alpha & \beta & \beta+\varepsilon \\
0 & 0 & 1 & 0 & \beta+\varepsilon & \alpha & \beta \\
0 & 0 & 0 & 1 & \beta & \beta+\varepsilon & \alpha
\end{array}\right)
$$

Each point in $\mathcal{A}_{\text {(VII) }}$ will be referred to using the number of its column in the above matrix, and subsets of $\mathcal{A}_{(\mathrm{VII})}$ will be denoted by simply enumerating the points they contain. Since $\alpha, \beta$, and $\varepsilon$ satisfy the system of inequalities given above, the following subsets of $\mathcal{A}_{(\mathrm{VII})}$ are precisely the 3-dimensional faces of a triangulation of $\mathcal{A}_{(\mathrm{VII})}$ :

1567, 1236, 1347, 1245, 1367, 1457, 1256, 2356, 3467, 2457.

This triangulation, thereafter referred to as triangulation (VII), is sketched in Fig. 2. The vector $u$ shown in this figure is obtained by scaling the sum of the three basis vectors by a positive coefficient. In the right side of the figure, only the following faces of (VII) have been represented as solid lines: 123, $124,134,236,347,245,256,367,457$, and 567. The other faces are omitted for the sake of clarity. Note in particular that all the boundary edges of triangulation (VII) are shown except for 35,46 , and 27 . The intersection of 


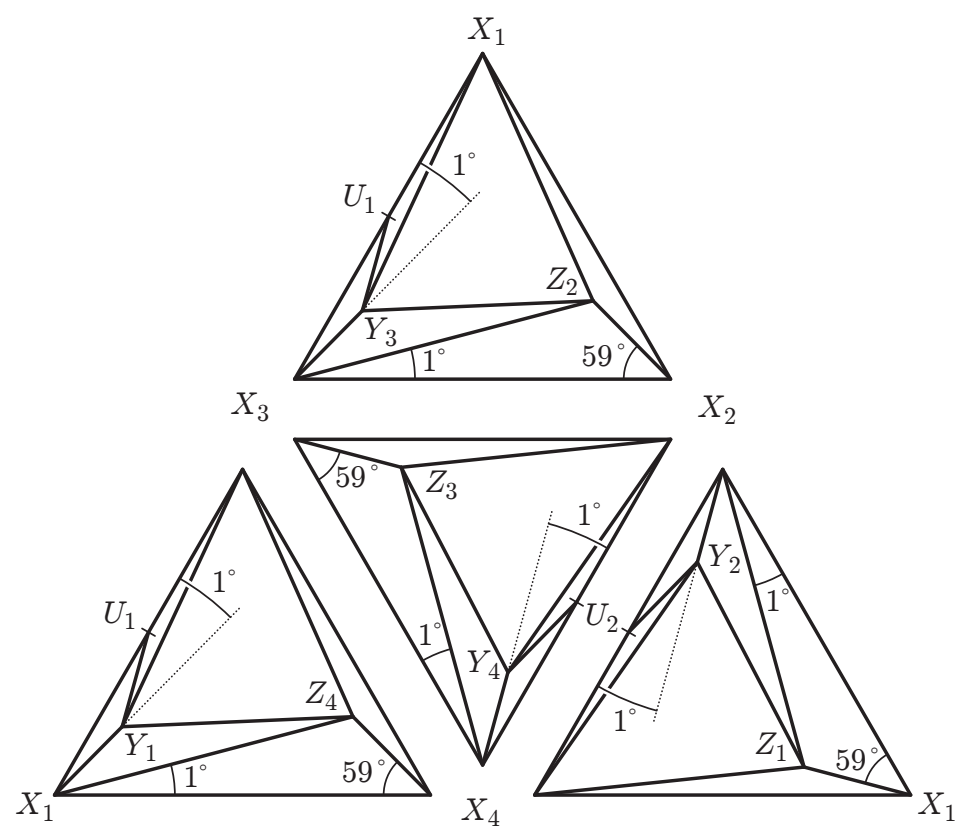

Fig. 3. The boundary faces of Rudin's triangulation

each face with the affine hull of triangle 234 is represented in the left of Fig. 2 , where the 2-dimensional interior faces correspond to dashed lines. One can see that the resulting intersection is precisely example (I). In particular, faces 136,147 , and 125 form a cycle with respect to the centroid of triangle 234 according to the definition in [7]. Hence, example (VII) is non-regular.

Theorem 3 can once again be invoked to prove that (VII) is 2-regular. Consider the triangulation obtained by flipping edge 26 in (VII), which amounts to replace the star of 26 by tetrahedra 1235 and 1356 together with their lower dimensional faces. This triangulation is regular and contains all the faces of (VII) except for those admitting edge $\{2,6\}$ as a subset. As the triangulation obtained by flipping edge $\{3,7\}$ in (VII) is regular as well and contains those seven missing faces, the 2-regularity of triangulation (VII) follows from theorem 3 .

The second 3-dimensional example studied in this paper is Rudin's triangulation [17]. This triangulation of a configuration of 14 points with no interior vertices was formulated originally as an example of a non-shellable triangulation. Its non-regularity therefore directly follows from its nonshellability. A comprehensive description of Rudin's triangulation can be found in [17]. The short description given here restricts to the facts needed to prove that this triangulation is 2-regular. The vertices of Rudin's triangulation will be denoted $X_{1}, \ldots, X_{4}, Y_{1}, \ldots, Y_{4}, Z_{1}, \ldots, Z_{4}, U_{1}$ and $U_{2}$ as in [17]. The distance between any two $X_{i}$ is equal to 1 , and all the vertices of the triangulation belong to the boundary of tetrahedron $\left\{X_{1}, X_{2}, X_{3}, X_{4}\right\}$. The 
placement of those vertices is therefore found by looking at the boundary faces of Rudin's triangulation, sketched in Fig. 3. Explicit coordinates can be obtained from the indications in Fig. 3, knowing that for all $i \in\{1,2,3,4\}$, the distance between $X_{i}$ and $Y_{i}$ is equal to $\sin \left(60^{\circ}\right) \sin \left(1^{\circ}\right) / \sin \left(61^{\circ}\right)$. Note that the angles in this figure have been scaled up or down for the sake of clarity, as vertices $Y_{i}$ and $Z_{i}$ are very close to the edges of the tetrahedron. In [17], Rudin classifies the 41 tetrahedra of her triangulation in 11 categories numbered (1) to (11), and uses an index $i \in\{1,2,3,4\}$ in order to refer to individual tetrahedra within each category. All categories contain four tetrahedra except for category (5) that only contains one tetrahedron. Rather than listing the tetrahedra here, references will be made to those categories and indices instead.

For $k \in\{1,2\}$, call $w_{k}$ the height function on the vertex set of Rudin's triangulation that maps $X_{k}$ and $X_{k+2}$ to 12 , the other two $X_{i}$ to $10^{\prime} 000, U_{k}$ to 11 , the four $Z_{i}$ to 0 and all the other vertices to 10 . Using formal calculations with the exact vertex coordinates from [17], one can check that $r\left(w_{k}\right)$ contains category (1), (2), (3), (4) and (11) tetrahedra for $i \notin\{k, k+2\}$, category (6), (7), (8), (9), and (10) tetrahedra for $i \in\{k, k+2\}$, and tetrahedron (5). As a consequence, Rudin's triangulation is a subset of $r\left(w_{1}\right) \cup r\left(w_{2}\right)$ and according to theorem 3, it is 2-regular. This shows in particular that, unlike regular subdivisions, 2-regular subdivisions are not necessarily shellable. Note that the height functions $w_{1}$ and $w_{2}$ have been found by running the simplex method with a set of constraints following the description in [23] and an ad-hoc objective function. The obtained optimal solution has then been modified to simpler figures and formal calculations have been used to check that the resulting height functions were still admissible.

\section{Bounds on $\delta$ over a class of subdivisions}

All the point configurations underlying the examples described in the previous section have some symmetry. The basic idea behind the construction of those examples is now synthesized and generalized to arbitrary point configurations. Upper bounds on the smallest value of $k$ so that such subdivisions are $k$-regular are subsequently given. For any subdivision $S$ of a point configuration $\mathcal{A}$, the set of all the faces of $S$ that lie within the relative boundary of $\operatorname{conv}(\mathcal{A})$ will be denoted by $b(S)$. Now, for any two subdivisions $S$ and $S^{\star}$ of possibly distinct point configurations, consider the set:

$$
\Pi\left(S, S^{\star}\right)=\left\{s \cup s^{\star}:\left(s, s^{\star}\right) \in b(S) \times b\left(S^{\star}\right)\right\}
$$

The examples described in section 3 have the following common feature: they can be built from two regular subdivisions $R$ and $R^{\star}$ by considering the whole of $R^{\star}$ together with additional faces in $\Pi\left(R, R^{\star}\right)$. This construction can be formalized as follows for arbitrary point configurations:

Definition 2. Let $\mathcal{A}$ and $\mathcal{A}^{\star}$ be disjoint point configurations whose convex hulls have non-disjoint relative interiors. Call $\kappa\left(\mathcal{A}, \mathcal{A}^{\star}\right)$ the set of all the 
subdivisions $S \in \omega\left(\mathcal{A} \cup \mathcal{A}^{\star}\right)$ so that there exist $R \in \rho(\mathcal{A})$ and $R^{\star} \in \rho\left(\mathcal{A}^{\star}\right)$ satisfying $S \backslash R^{\star} \subset \Pi\left(R, R^{\star}\right)$.

One can see that all the subdivisions studied in the previous section belong to some $\kappa\left(\mathcal{A}, \mathcal{A}^{\star}\right)$. For subdivisions (I) to (VI), $\mathcal{A}^{\star}$ is the set of the interior vertices of the subdivision. In the case of triangulation (VII), $\mathcal{A}^{\star}$ is constituted of vertices $1,5,6$, and 7 and for Rudin's triangulation $\mathcal{A}^{\star}=\left\{Z_{1}, Z_{2}, Z_{3}, Z_{4}\right\}$. In every case, $\mathcal{A}$ is obtained by removing the elements of $\mathcal{A}^{\star}$ from the vertex set of the subdivision. Theorem 4 focuses on a proper subset of $\kappa\left(\mathcal{A}, \mathcal{A}^{\star}\right)$. This subset does not contain all of above subdivisions, but is interesting as a standalone set of examples. Moreover the proof of theorem 4 gives a simpler version of the arguments that will be used to prove theorem 5 . In the proofs of both theorems, for any affine subspace $E$ of $\mathbb{R}^{n}$ the orthogonal projection from $\mathbb{R}^{n}$ onto $E$ is denoted by $\pi_{E}$.

Theorem 4. Let $\mathcal{A} \subset \mathbb{R}^{n}$ and $\mathcal{A}^{\star} \subset \mathbb{R}^{n}$ be disjoint point configurations whose convex hulls have non-disjoint relative interiors. For every subdivision $S$ of $\mathcal{A} \cup \mathcal{A}^{\star}$ so that $S \subset \Pi\left(t(\mathcal{A}), t\left(\mathcal{A}^{\star}\right)\right), \delta(S) \leq \min \left(\operatorname{dim}(\mathcal{A}), \operatorname{dim}\left(\mathcal{A}^{\star}\right)\right)$.

Proof. It will be assumed throughout the proof that 0 belongs to the relative interiors of both $\operatorname{conv}(\mathcal{A})$ and $\operatorname{conv}\left(\mathcal{A}^{\star}\right)$. This requirement can be achieved without loss of generality by using, if needed, a translation of $\mathcal{A} \cup \mathcal{A}^{\star}$. The affine hulls of $\mathcal{A}$ and $\mathcal{A} \cup \mathcal{A}^{\star}$ will be denoted by $E$ and $F$ respectively. Observe that those affine hulls are linear subspaces of $\mathbb{R}^{n}$. Denote by $k$ the dimension of $E$ and by $d$ the dimension of $F$. One can further assume without loss of generality that $n=d+k$. Call $H$ the orthogonal complement of $F$ in $\mathbb{R}^{n}$ and $\eta$ a linear bijection from $E$ onto $H$. Consider the map:

$$
\begin{aligned}
\phi: \mathcal{A} & \rightarrow \mathbb{R}^{d+k}, \\
a & \mapsto \mid \begin{array}{ll}
a+\eta(a) & \text { if } a \in \mathcal{A}, \\
a & \text { if } a \in \mathcal{A}^{\star} .
\end{array}
\end{aligned}
$$

The set $\phi(\mathcal{A})$ is a point configuration in $\mathbb{R}^{n}$ whose image by $\pi_{F}$ is exactly $\mathcal{A}$. Denote by $p$ the convex hull of $\phi(\mathcal{A})$ and observe that $\left(p, \pi_{F}\right)$ is a polytope projection. It is now proven that for all $s \in \Pi\left(t(\mathcal{A}), t\left(\mathcal{A}^{\star}\right)\right)$, the convex hull of $\phi(s)$ is a face of $p$.

Let $s$ be an element of $\Pi\left(t(\mathcal{A}), t\left(\mathcal{A}^{\star}\right)\right)$. Note that $\mathcal{A} \cap s$ is a boundary face of $t(\mathcal{A})$. As a consequence there exists an affine map $\psi: E \rightarrow \mathbb{R}$ that projects $\mathcal{A} \cap s$ onto $\{0\}$ and the rest of $\mathcal{A}$ into $]-\infty, 0[$. As the convex hull of $\mathcal{A}$ contains 0 in its relative interior, one has $\psi(0)<0$. A similar argument can be used to find a map $\psi^{\star}: F \rightarrow \mathbb{R}$ that projects $\mathcal{A}^{\star} \cap s$ onto $\{0\}$ and the rest of $\mathcal{A}^{\star}$ into $]-\infty, 0\left[\right.$. As 0 lies within the relative interior of $\operatorname{conv}\left(\mathcal{A}^{\star}\right)$, one can further require that $\psi(0)=\psi^{\star}(0)$. Now consider the affine map:

$$
\mu=\psi^{\star} \circ \pi_{F}+\left(\psi-\psi^{\star}\right) \circ \eta^{-1} \circ \pi_{H} .
$$

One can see that $\mu \circ \phi$ coincides with $\psi$ on $\mathcal{A}$ and with $\psi^{\star}$ on $\mathcal{A}^{\star}$. Following this, $\mu$ projects all the elements of $\phi(s)$ onto $\{0\}$ and the rest of $\phi\left(\mathcal{A} \cup \mathcal{A}^{\star}\right)$ into ] $-\infty, 0[$. As a consequence, the convex hull of $\phi(s)$ is a face of $p$. 
As subdivision $S$ is found as a subset of $\pi\left(t(\mathcal{A}), t\left(\mathcal{A}^{\star}\right)\right)$, it therefore necessarily belongs to $\omega\left(p, \pi_{F}\right)$. Moreover, as $n-d$ is equal to $\operatorname{dim}(\mathcal{A})$, then $\delta(S) \leq \operatorname{dim}(\mathcal{A})$. The statement of the theorem being symmetrical in $\mathcal{A}$ and $\mathcal{A}^{\star}$, the result follows.

One can see that subdivisions (I), (II), and (IV) are found as subsets of $\Pi\left(t(\mathcal{A}), t^{\star}\left(\mathcal{A}^{\star}\right)\right)$ for well chosen point configurations $\mathcal{A}$ and $\mathcal{A}^{\star}$. Indeed, if one takes $\mathcal{A}=\{1,2,6\}$ and $\mathcal{A}^{\star}=\{3,4,5\}$ in the case of triangulation (I), then this triangulation is a subset of $\Pi\left(t(\mathcal{A}), t^{\star}\left(\mathcal{A}^{\star}\right)\right)$. Subdivisions (II) and (IV) are also found as subsets of $\Pi\left(t(\mathcal{A}), t^{\star}\left(\mathcal{A}^{\star}\right)\right)$ if in those two cases $\mathcal{A}=\{1,2,7,8\}$ and $\mathcal{A}^{\star}=\{3,4,5,6\}$. It follows that theorem 4 can be used to prove that those three subdivisions are 2-regular, thus providing tight bounds on $\delta$. Triangulation (VII) is also found as a subset of $\Pi\left(t(\mathcal{A}), t^{\star}\left(\mathcal{A}^{\star}\right)\right)$ if $\mathcal{A}=\{1,2,3,7\}$ and $\mathcal{A}^{\star}=\{4,5,6\}$. Note that $\mathcal{A}^{\star}$ then is 2-dimensional. Theorem 4 can therefore be invoked once more to prove that triangulation (VII) is 2-regular, which also provides a tight bound on $\delta$.

Example (III) is not a subset of any $\Pi\left(t(\mathcal{A}), t^{\star}\left(\mathcal{A}^{\star}\right)\right)$ as its central pentagon cannot be decomposed as the union of two of its faces. As a consequence, theorem 4 cannot be used in this case. Triangulation (V) does not satisfy to the requirements of theorem 4 either. Indeed, assume that the vertex set of this triangulation is partitioned into subsets $\mathcal{A}$ and $\mathcal{A}^{\star}$, and that vertex 10 belongs to $\mathcal{A}$. In order for 10 to belong to the boundary of $\operatorname{conv}(\mathcal{A})$, one of the three sets $\{1,4,9\},\{2,5,7\}$, and $\{3,6,8\}$ has to be disjoint of $\mathcal{A}$. Say $\{1,4,9\} \cap \mathcal{A}=\emptyset$ which, by symmetry, can be assumed without loss of generality. Then, at least one point in $\{2,5,7\}$ and one point in $\{3,6,8\}$ must be in $\mathcal{A}^{\star}$, otherwise the convex hull of $\mathcal{A}$ contains either 2 or 3 in its interior. As a consequence, vertex 1 is necessarily in the interior of $\mathcal{A}^{\star}$. This proves that there exists no partition $\left(\mathcal{A}, \mathcal{A}^{\star}\right)$ of the vertex set of triangulation $(\mathrm{V})$ so that this triangulation is found as a subset of $\Pi\left(t(\mathcal{A}), t^{\star}\left(\mathcal{A}^{\star}\right)\right)$. Theorem 4 therefore cannot be used in this case either. The next theorem provides slightly larger bounds on $\delta$ than theorem 4 , but those hold over the whole of $\kappa\left(\mathcal{A}, \mathcal{A}^{\star}\right)$ :

Theorem 5. Let $\mathcal{A} \subset \mathbb{R}^{n}$ and $\mathcal{A}^{\star} \subset \mathbb{R}^{n}$ be disjoint point configurations whose convex hulls have non-disjoint relative interiors. For every subdivision $S$ in $\kappa\left(\mathcal{A}, \mathcal{A}^{\star}\right), \delta(S) \leq \min \left(\operatorname{dim}(\mathcal{A}), \operatorname{dim}\left(\mathcal{A}^{\star}\right)\right)+1$.

Proof. Respectively call $E$ and $F$ the affine hulls of $\mathcal{A}$ and $\mathcal{A} \cup \mathcal{A}^{\star}$. Further denote $k=\operatorname{dim}(E)$ and $d=\operatorname{dim}(F)$. One can assume without loss of generality that $n=d+k+1$. It is also possible to require that 0 belongs to the relative interiors of both $\operatorname{conv}(\mathcal{A})$ and $\operatorname{conv}\left(\mathcal{A}^{\star}\right)$ by using, if needed, a translation of $\mathcal{A} \cup \mathcal{A}^{\star}$. Following this, $E$ and $F$ are linear subspaces of $\mathbb{R}^{n}$. The orthogonal complement of $F$ in $\mathbb{R}^{n}$ will be decomposed as the orthogonal direct sum of a $k$-dimensional linear space $H$ with the linear span of a unit vector $u \in \mathbb{R}^{n}$. Let $\eta$ be a linear bijection from $E$ onto $H$. Let $R$ and $R^{\star}$ be regular subdivisions of $\mathcal{A}$ and $\mathcal{A}^{\star}$ respectively. Call $w: \mathcal{A} \rightarrow \mathbb{R}$ and $w^{\star}: \mathcal{A}^{\star} \rightarrow \mathbb{R}$ two height functions so that $R=r(w)$ and $R^{\star}=r\left(w^{\star}\right)$. 
Let $\beta$ be any real number. Consider the map:

$$
\begin{aligned}
\phi_{\beta}: \mathcal{A} & \rightarrow \mathbb{R}^{d+k}, \\
a & \mapsto \mid \begin{array}{ll}
a+\eta(a)+w(a) u & \text { if } a \in \mathcal{A}, \\
a+\left[w^{\star}(a)+\beta\right] u & \text { if } a \in \mathcal{A}^{\star} .
\end{array}
\end{aligned}
$$

The set $\phi_{\beta}(\mathcal{A})$ then is a point configuration in $\mathbb{R}^{n}$ whose image by $\pi_{F}$ is exactly $\mathcal{A}$. Call $p_{\beta}$ the convex hull of $\phi_{\beta}(\mathcal{A})$ and observe that $\left(p_{\beta}, \pi_{F}\right)$ is a polytope projection. It will be shown as a first step that for all $s \in \Pi\left(R, R^{\star}\right)$, the convex hull of $\phi_{\beta}(s)$ is a face of $p_{\beta}$.

Let $s$ be an element of $\Pi\left(R, R^{\star}\right)$. Respectively call $L$ and $L^{\star}$ the affine hulls of $s \cap \mathcal{A}$ and $s \cap \mathcal{A}^{\star}$. As $s \cap \mathcal{A}$ lies within the relative boundary of $\operatorname{conv}(\mathcal{A})$, there exists an affine map $\psi: E \rightarrow \mathbb{R}$ that projects $\mathcal{A} \cap L$ onto $\{0\}$ and the rest of $\mathcal{A}$ into $]-\infty, 0[$. As 0 lies within the relative interior of $\operatorname{conv}(\mathcal{A})$, one necessarily has $\psi(0)<0$. With a similar argument, one finds an affine map $\psi^{\star}: F \rightarrow \mathbb{R}$ that projects $\mathcal{A}^{\star} \cap L^{\star}$ onto $\{0\}$ and the rest of $\mathcal{A}^{\star}$ into ] $-\infty, 0$ [. As 0 belongs to the relative interior of $\mathcal{A}^{\star}$ and as $\psi(0)<0$, it can further be required that $\psi(0)=\psi^{\star}(0)$.

Now observe that $\mathcal{A} \cap s$ is a face of $R$. There exists as a consequence an affine map $\xi: E \rightarrow \mathbb{R}$ so that $\xi(a)=w(a)$ for all $a \in \mathcal{A} \cap s$ and $\xi(a)<w(a)$ for $a \in \mathcal{A} \backslash s$. Since 0 does not belong to $L$, it is possible to find a linear functional $\zeta: E \rightarrow \mathbb{R}$ that coincides with $\xi$ on $L$. As $w^{\star}+\beta$ realizes $R^{\star}$, one can use the same trick to build a linear functional $\zeta^{\star}: F \rightarrow \mathbb{R}$ that coincides with $w^{\star}+\beta$ on $\mathcal{A}^{\star} \cap s$ and that further satisfies $\zeta^{\star}(a)<w^{\star}(a)+\beta$ for all $a \in\left(\mathcal{A}^{\star} \cap L^{\star}\right) \backslash s$. In the following of the proof, for any $x \in \mathbb{R}^{n}, \chi(x)$ denotes the scalar product of $x$ and $u$. Let $\theta$ be any positive real number. Consider the affine map:

$$
\mu_{\theta}=\left[\psi^{\star}+\theta \zeta^{\star}\right] \circ \pi_{F}+\left[\psi-\psi^{\star}+\theta\left(\zeta-\zeta^{\star}\right)\right] \circ \eta^{-1} \circ \pi_{H}-\theta \chi .
$$

Using the definitions of $\mu_{\theta}$ and $\phi_{\beta}$, one finds that $\mu_{\theta} \circ \phi_{\beta}$ coincides with $\psi+\theta(\zeta-w)$ on $\mathcal{A}$ and with $\psi^{\star}+\theta\left(\zeta^{\star}-w^{\star}-\beta\right)$ on $\mathcal{A}^{\star}$. As a first consequence, $\mu_{\theta} \circ \phi_{\beta}(s)=\{0\}$. Secondly, as $\theta>0, \mu_{\theta} \circ \phi_{\beta}$ projects $(\mathcal{A} \cap L) \backslash s$ and $\left(\mathcal{A}^{\star} \cap L^{\star}\right) \backslash s$ into $]-\infty, 0\left[\right.$. Moreover, as $\psi(\mathcal{A} \backslash L)$ and $\psi^{\star}\left(\mathcal{A}^{\star} \backslash L^{\star}\right)$ both are subsets of ] $-\infty, 0[$, there necessarily exists a real number $\alpha>0$ so that if $0<\theta<\alpha$, then $\mu_{\theta}$ projects $\mathcal{A} \backslash L$ and $\mathcal{A}^{\star} \backslash L^{\star}$ into $]-\infty, 0[$. As a consequence, for all $\theta \in] 0, \alpha\left[, \mu_{\theta}\right.$ maps all the elements of $\phi_{\beta}(s)$ to 0 and the rest of $\phi_{\beta}\left(\mathcal{A} \cup \mathcal{A}^{\star}\right)$ to negative numbers. It follows that the convex hull of $\phi_{\beta}(s)$ is a face of $p_{\beta}$. Note that this property holds for all $\beta \in \mathbb{R}$.

Now let $s \in R^{\star}$. According to the definition of regular subdivisions, there exists an affine map $\xi^{\star}: F \rightarrow \mathbb{R}$ that coincides with $w^{\star}$ on $s$ and that is smaller than $w^{\star}$ on the rest of $\mathcal{A}^{\star}$. Consider the affine map:

$$
\psi^{\star}=\xi^{\star} \circ \pi_{F}-\chi+\beta .
$$

Observe that $\psi^{\star} \circ \phi_{\beta}$ coincides with $\xi^{\star}-w+\beta$ on $\mathcal{A}$ and with $\xi^{\star}-w^{\star}$ on $\mathcal{A}^{\star}$. Call $\alpha_{s}^{\star}$ the minimum of $\xi^{\star}-w$ on $\mathcal{A}$. One can see that if $\beta$ is larger than $-\alpha_{s}^{\star}$ then $\psi^{\star}$ projects $\phi_{\beta}(s)$ onto $\{0\}$ and the rest of $\phi_{\beta}\left(\mathcal{A} \cup \mathcal{A}^{\star}\right)$ into 
] $0,+\infty\left[\right.$. Following this, whenever $\beta>-\alpha_{s}$, the convex hull of $\phi_{\beta}(s)$ is a face of $p_{\beta}$. As the intersection $\left.\cap_{s \in R^{\star}}\right]-\alpha_{s}^{\star},+\infty$ [ is non-empty, there exists $\beta \in \mathbb{R}$ so that for all $s \in R^{\star}$, the convex hull of $\phi_{\beta}(s)$ is a face of $p_{\beta}$.

This proves the existence of a number $\beta \in \mathbb{R}$ so that all the subdivisions found a subsets of $R^{\star} \cup \Pi\left(R, R^{\star}\right)$ necessarily belong to $\omega\left(p_{\beta}, \pi_{E}\right)$. Since this property holds for every pair $\left(R, R^{\star}\right)$ in $\rho(\mathcal{A}) \times \rho\left(\mathcal{A}^{\star}\right), \delta$ admits $\operatorname{dim}\left(\mathcal{A}^{\star}\right)+1$ as an upper bound over the whole of $\kappa\left(\mathcal{A}, \mathcal{A}^{\star}\right)$. It will now be shown that the same bound holds on $\delta$ over $\kappa\left(\mathcal{A}^{\star}, \mathcal{A}\right)$. This will prove by symmetry that $\delta$ is never larger than $\operatorname{dim}(\mathcal{A})+1$ over $\kappa\left(\mathcal{A}, \mathcal{A}^{\star}\right)$. Observe that in order to obtain this result, one only needs to show that all the subdivisions found as subsets of $R \cup \Pi\left(R, R^{\star}\right)$ necessarily belong to $\omega\left(p_{\beta}, \pi_{E}\right)$ for some $\beta \in \mathbb{R}$. Let $s$ be a face of $R$ and $\xi: F \rightarrow \mathbb{R}$ an affine map that coincides with $w$ on $s$ and so that $\xi(a)<w(a)$ for all $a \in \mathcal{A} \backslash s$. Consider the affine map:

$$
\psi=\xi \circ \pi_{F}-\chi .
$$

The map $\psi \circ \phi_{\beta}$ coincides with $\xi-w$ on $\mathcal{A}$ and with $\xi-w^{\star}-\beta$ on $\mathcal{A}^{\star}$. Call $\alpha_{s}$ the minumum of $\xi-w^{\star}$ on $\mathcal{A}^{\star}$. If $\beta$ is smaller than $\alpha_{s}$ then $\psi$ projects $\phi_{\beta}(s)$ onto $\{0\}$ and the rest of $\phi_{\beta}\left(\mathcal{A} \cup \mathcal{A}^{\star}\right)$ into $] 0,+\infty[$. As a consequence, for all $\beta<\alpha_{s}$, the convex hull of $\phi_{\beta}(s)$ is a face of $p_{\beta}$. Let $\beta$ be smaller than $\alpha_{s}$ for all $s \in R$. In this case, the convex hull of $\phi_{\beta}(s)$ is a face of $p_{\beta}$ for all $s \in R$. One therefore obtains that all the subdivisions found as subsets of $R \cup \Pi\left(R, R^{\star}\right)$ necessarily belong to $\omega\left(p_{\beta}, \pi_{E}\right)$ for some small enough $\beta$. As this holds for every pair $\left(R, R^{\star}\right)$ in $\rho(\mathcal{A}) \times \rho\left(\mathcal{A}^{\star}\right), \delta$ is never larger than $\operatorname{dim}\left(\mathcal{A}^{\star}\right)+1$ over $\kappa\left(\mathcal{A}^{\star}, \mathcal{A}\right)$, and the result follows.

Observe that the bounds provided by theorem 5 are not tight. Indeed, using this theorem one can prove at best that Rudin's triangulation is 4-regular and that the other examples of section 3 are 3 -regular. While better bounds were obtained from theorem 3 , it was at the expense of verifying rather strong requirements specific to each subdivision. Theorems 4 and 5 do not have any such requirements and provide general bounds over whole sets of subdivisions.

\section{Discussion}

The notion of $k$-regularity sheds a new light on the structure of the refinement posets of point configurations. In particular, it makes it possible to identify a connected subgraph in the flip-graph of a point configuration. In this section, a few questions related to $k$-regularity and flip-graph connectivity are formulated and a conjecture is stated.

Let $\mathcal{A}$ be a point configuration and $S \in \omega(\mathcal{A})$ a subdivision. It is natural to ask about the smallest integer $k$ so that $S$ is $k$-regular. As mentioned in section $2, S$ is necessarily $(n-d-1)$-regular, where $n$ is the cardinality of $\mathcal{A}$ and $d$ the dimension of its affine hull. While this provides a bound on $\delta$ that holds in general, it is a very loose one in the case of large point configurations 
as it depends on $n$. In section 4 , bounds are given on $\delta$ that do not depend on the size of the underlying point configuration. Those bounds, however only hold for a particular class of subdivisions and are not necessarily tight. The following first question therefore still has to be addressed:

Question 1. For any $d>1$, what is the smallest integer $k$ so that if a point configuration is $d$-dimensional then all its subdivisions are $k$-regular?

The study made in section 3 of several non-regular subdivisions known from the literature suggests that the subdivisions of 2-dimensional point configurations are always 2-regular. Although the 3-dimensional examples that have been reviewed turn out to be 2-regular as well, it seems unlikely that this observation carries over in general to 3 -dimensional point configurations. It is natural, however, to formulate the following conjecture in an attempt to answer question 1, at least partially:

Conjecture 1. For any $d$-dimensional point configuration $\mathcal{A}, \omega(\mathcal{A}) \subset \rho_{d}(\mathcal{A})$.

Now let $\mathcal{A}$ be a point configuration. As shown in $[9,10]$, the graph $\gamma_{1}(\mathcal{A})$ is connected and according to theorem $2, \gamma_{2}(\mathcal{A})$ is connected as well. In particular, if all the triangulations of $\mathcal{A}$ are 2-regular then $\gamma(\mathcal{A})$ is connected. This shows that question 1 is directly relevant to the problem of flip-graph connectivity. The status of $\gamma_{k}(\mathcal{A})$ regarding connectivity is still to be elucidated for $k>2$, and the following second question arises:

Question 2. What is the largest integer $k$ so that the subgraph induced by $k$-regular triangulations in the flip-graph of any point configuration is connected?

Observe that questions 1 and 2 decompose the problem of flip-graph connectivity into two steps. For example, if conjecture 1 holds and $\gamma_{3}(\mathcal{A})$ is connected for any point configuration $\mathcal{A}$, then all the 3 -dimensional point configurations necessarily have a connected flip-graph. Questions 1 and 2 are also relevant separately, though, and each of them could provide insights in the structure of the refinement poset of point configurations.

Finally, it has been proven in section 3 that Rudin's triangulation is 2-regular. As a direct consequence, the natural implication between regularity and shellability does not hold in general for $k$-regularity. It is therefore relevant to study the opposite implication:

Question 3. What is the smallest integer $k$ so that all the shellable subdivisions are $k$-regular?

\section{References}

[1] V. Alexeev, Complete moduli in the presence of semiabelian group action, Ann. of Math. 155, 611-708 (2002) 
[2] M. Azaola and F. Santos, The graph of triangulations of a point configuration with $d+4$ vertices is 3-connected, Discrete Comput. Geom. 23, 4, 489-536 (2000)

[3] H. J. Baues, Geometry of loop spaces and the cobar construction, Mem. Am. Math. Soc. 25, 99-124 (1980)

[4] L. J. Billera and B. Sturmfels, Fiber polytopes, Ann. of Math. 135, 527-549 (1992)

[5] L. J. Billera, M. M. Kapranov and B. Sturmfels, Cellular strings on polytopes, Proc. Amer. Math. Soc. 122, 2, 549-555 (1994)

[6] J. A. De Loera, J. Rambau, F. Santos, Triangulations: structures for algorithms and applications, Algorithms and Computation in Mathematics 25, Springer (2010)

[7] H. Edelsbrunner, An acyclicity theorem for cell complexes in $d$ dimension, Combinatorica 10, 3, 251-260 (1990)

[8] H. Edelsbrunner and N. R. Shah, Incremental topological flipping works for regular triangulations, Algorithmica 15, 3, 223-241 (1996)

[9] I. M. Gel'fand, M. M. Kapranov and A. V. Zelevinsky, Discriminants of polynomials of several variables and triangulations of Newton polyhedra, Leningrad Math. J. 2, 449-505 (1990)

[10] I. M. Gel'fand, M. M. Kapranov and A. V. Zelevinsky, Discriminants, resultants, and multidimensional determinants, in: Mathematics: Theory \& Applications, Birkhäuser, Boston, 1994

[11] C. L. Lawson, Software for $C^{1}$ interpolation, in Mathematical Software III (John Rice, ed.), Academic Press, New York (1977)

[12] L. Pournin and Th. M. Liebling, Constrained paths in the flip-graph of regular triangulations, Comput. Geom. 37, 2, 134-140 (2007)

[13] L. Pournin and Th. M. Liebling, From spheres to spheropolyhedra: generalized Distinct Element Methodology and algorithm analysis, in Research Trends in Combinatorial Optimization (W. Cook, L. Lovász, and J. Vygen eds.), 347363, Springer, Berlin (2009)

[14] J. Radon, Mengen konvexer Körper, die einen gemeinsamen Punkt enthalten, Math. Ann. 83, 113-115 (1921)

[15] J. Rambau and G. M. Ziegler, Projections of polytopes and the generalized Baues conjecture, Discrete Comput. Geom. 16, 3, 215-237 (1996)

[16] J. Rambau, On a generalization of Schönhardt's polyhedron, in Combinatorial and Computational Geometry (J. E. Goodman, J. Pach, and E. Welzl, eds.), MSRI publications 52, 501-516 (2005)

[17] M. E. Rudin, An unshellable triangulation of a tetrahedron, B. Am. Math. Soc. 64, 3, 90-91 (1958)

[18] F. Santos, A point set whose space of triangulations is disconnected, J. Am. Math. Soc. 13, 611-637 (2000)

[19] F. Santos, On the refinements of a polyhedral subdivision, Collect. Math. 52, 3, 231-256 (2001)

[20] F. Santos, Non-connected toric Hilbert schemes, Math. Ann. 332, 645-665 (2005)

[21] F. Santos, Geometric bistellar flips: the setting, the context and a construction, Proceedings of the ICM 2006, III, 931-962 (2006)

[22] E. Schönhardt, Über die Zerlegung von Dreieckspolyedern in Tetraeder, Math. Ann. 89, 309-312 (1927)

[23] F. Takeuchi, Nonregular triangulations, view graphs of triangulations, and linear programming duality, Lecture Notes in Computer Science 2098, 330-338 (2001) 